\title{
Behavioral Modeling of Power Semiconductors in Modelica
}

\author{
Patrick Denz \\ Vorarlberg Univ. of Appl. Sc. \\ Dornbirn/Austria \\ Patrick.Denz@omicron.at
}

\author{
Thomas Schmitt \\ Modelon $\mathrm{GmbH}$ \\ Munich/Germany \\ Thomas.Schmitt@modelon.com
}

\author{
Markus Andres \\ Modelon $\mathrm{GmbH}$ \\ Munich/Germany \\ Markus.Andres@modelon.com
}

\begin{abstract}
This paper introduces behavioral (macro) models of power semiconductors, i.e. diodes, MOSFETs and IGBTs, being part of a library for simulating power electronics utilized, e.g. in electrified powertrains of either hybrid electric vehicles (HEV) or purely battery electric vehicles $(B E V)$. The models consider static, dynamic (switching mode) and thermal effects and in most cases can be fully parameterized solely on the basis of characteristic curves and parameters specified in datasheets. The main purpose of behavioral models is an accurate representation of the semiconductor signals to, e.g. calculate the overall losses. The MOSFET models are verified in simulations with various test circuits and are validated with measurement data provided by a company developing electric drive systems. Furthermore, the arising numerical problems are discussed and possible solutions are provided on how to modify the models in order to use them in e.g. system simulation.
\end{abstract}

Keywords: power electronics, power semiconductors, macro modeling, behavioral modeling, numerical performance

\section{Introduction}

In practice, models of electric powertrains - consisting of at least a high-voltage battery, an inverter and an electric machine - have to answer questions regarding lifetime, maximum driving range, temperature development or overall efficiency. One of the most important and challenging tasks is to provide models that can be parameterized easily and simulated fast and robust, i.e. numerically stable.

Models of power electronic components are available in different degrees of complexity in freely- and commercially distributed libraries. When needed for industrial use, ideal models are often not accurate enough, whereas physical ones cannot be parameterized with standard datasheets and are rather suited for the field of research. Moreover, such models are often solely available in a specific level of detail which either results in unreasonable simulation times in case of very detailed models or a lack of information in case of e.g. ideal models. In [6] different modeling techniques of the switch models available in the Modelica Standard Library (MSL) are discussed.

A trade-off between these ideal and micro modeling techniques is a macro modeling approach called behavioral modeling, which was first introduced for power semiconductors in [8] and is further developed at Modelon GmbH. The idea of this technique is to describe the component's behavior via characteristic curves and parameters provided in datasheets. Thus, on the one hand behavioral models of MOSFETs can be parameterized solely on the basis of datasheets and on the other hand, the models behave as specified by the manufacturer under nominal conditions. In case of IGBTs due to their internal semiconductor structure, the occurring tail current has to be measured in advance. Moreover, in trench/field-stop IGBTs due to the additional field-stop layer added to the semiconductor structure the model developed in [8] is not valid anymore and has to be modified.

Since behavioral models provide detailed switching slopes, the simulation performance is totally unacceptable if such models are used to simulate, e.g. the driving range of an electric vehicle. Hence, some possibilities are discussed on how to use such models to derive table based models that store solely the information needed for a specific simulation task.

\section{Behavioral Modeling of Diodes}

In the Modelica Standard Library (MSL) several different models of diodes are available (refer to [6]). The ideal diodes are modeled using parameterized curve descriptions, whereas the physical ones are described 
by the well-known Shockley equation. These models are perfectly suited for, e.g. circuit analysis. However, if conduction- and switching losses of a specific diode are of interest none of these models can be used since they neither include the appropriate equations describing the transient (switching) behavior nor cannot be parameterized using datasheets. Behavioral models provide the means to make use of datasheet values to model the static- and dynamic behavior.

\subsection{Static Model}

In the simplest case, the static model of a diode can be described as depicted in Figure 1. The current through the diode is measured using a current sensor and serves as input signal to a table which stores the forward characteristic $V_{f}=f\left(I_{f}\right)$ specified in the datasheet. The corresponding forward voltage drop is fed into a signal-controlled voltage source. The additional ideal reverse blocking diode ensures that the current solely flows in forward direction.

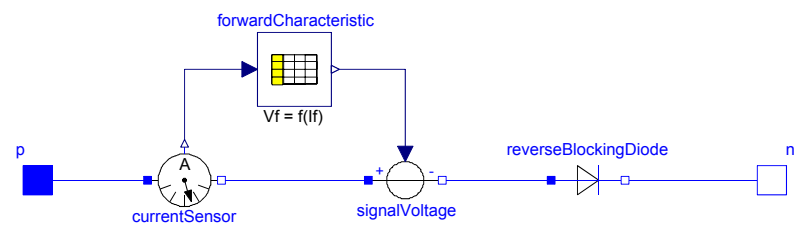

Figure 1: Static model of a diode: $V_{f}=f\left(I_{f}\right)$

Usually datasheets provide the diode's forward characteristic not only as a function of the forward current $I_{f}$ but also of the temperature $T$. Hence, the model has to be modified as depicted in Figure 2.

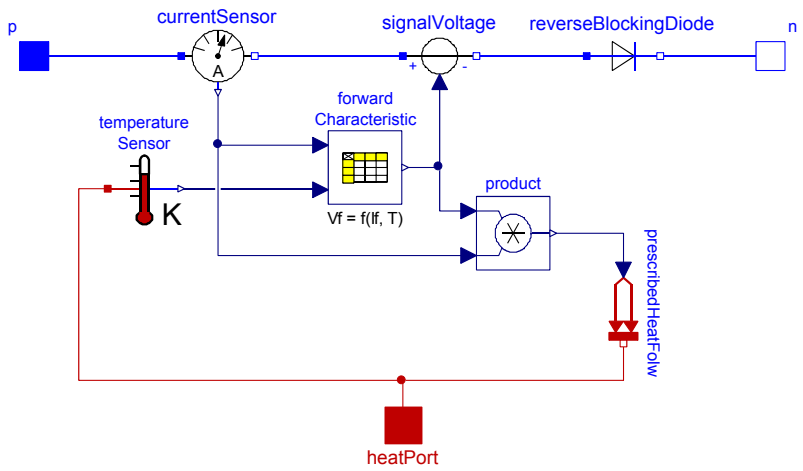

Figure 2: Static model of a diode: $V_{f}=f\left(I_{f}, T\right)$

The losses generated while the diode is conducting are nothing else but a thermal heat flow which will result in a certain temperature depending on the thermal network connected to the heat port.

\subsection{Dynamic Model (Reverse Recovery Ef- fect)}

The dynamics of a diode occur due to its junction- and diffusion-capacitance. An approach of modeling these capacitances is given in [8]. The main problem is that the capacitance values depend on parameters that are not available in standard datasheets. As the dynamics of a diode in terms of switching losses are mainly dependent on the reverse recovery effect, an approach has been followed which was published in [3].

\section{Behavioral Modeling of Power- MOSFETs}

In order to understand the behavioral model of a power-MOSFET its structure will be discussed briefly. After introducing the models its modes of operation will be verified using different test circuits. Finally, the model is validated with measurement results provided by a company.

\subsection{Power-MOSFET Structure and its Modes of Operation}

By means of the MOSFET's semiconductor structure, the different modes of operation shall now be analyzed. Based on that, a static model can be developed. In Figure 3, the structure of a vertical power-MOSFET is illustrated.

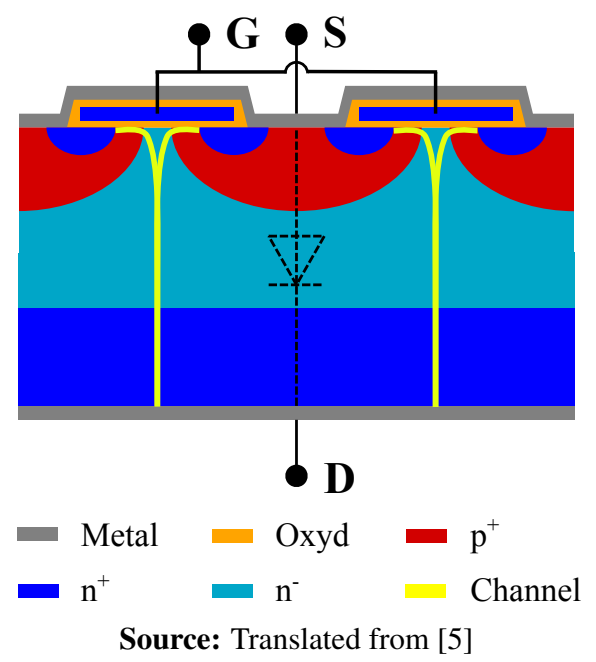

Figure 3: Structure of a power-MOSFET

It is first supposed that a positive gate-source voltage is applied, i.e. a conducting channel between drain and source arises. Now, the MOSFET operates in on-state. Thus, if a positive drain-source voltage is applied (on-state forward conduction mode), 
current flows from drain to source, which represents the MOSFET's first quadrant operation. Since the conducting channel is not a pn-junction but more of a voltage-controlled resistance, current can also flow from source to drain if a negative drain-source voltage is applied (on-state reverse conduction). This was not considered in the model developed in [8] and will be added to the model demonstrated in this paper. In the modified version of the MOSFET, the characteristic curves in the first quadrant are mirrored into the third quadrant. The only difference in third quadrant application is an additional parasitic diode - the body diode - between drain and source. If the current in reverse direction is high enough to cause a voltage drop equal to the body diode's threshold voltage, the body diode starts conducting and provides an additional current path. A more detailed description of these reverse conduction modes can be found in [9, p. $61 \mathrm{ff}$.]. In order to analyze the off-state modes, the gate-source voltage is supposed to be zero meaning that the conduction channel does not arise. Still, the body diode can conduct current in reverse direction. In forward direction, no current can flow. In summary, the discussed modes of operation are listed below.

Mode 1: $\quad$ on-state forward conduction

Mode 2: $\quad$ on-state reverse conduction

Mode 3: $\quad$ on-state reverse conduction with

body diode forward biased

Mode 4: $\quad$ off-state reverse conduction

Mode 5: off-state forward blocking

\subsection{Power-MOSFET Model}

Based on the modes of operation, a behavioral model of the power-MOSFET is developed in the following. First, a static model is derived, which afterwards is extended to cover dynamics and temperature dependency.

\subsubsection{Static Model}

In order to describe the static behavior of a MOSFET, the modes of operation discussed before have to be realized in a model structure. As depicted in Figure 4, the transfer behavior and therefore the MOSstructure is modeled by a voltage sensor measuring the applied gate-source voltage. This voltage signal is the input to a table which stores the transfer characteristic $I_{d}=f\left(V_{g s}\right)$ specified in the datasheet. The table's output is the maximum current, which can flow due to the applied gate-source voltage. This current value is the input to the signal-controlled current sources. Since the current that flows through the component is determined by the external load circuit, e.g. by an inductive load, each current source has an ideal diode in parallel ensuring that the current that is not drawn by the load can free-wheel through these diodes. In Figure 4 the current paths in the different modes of operation are marked with arrows. In mode 1 , the current flows through the resistor RonFw, which represents the conducting channel. Another ideal diode is connected in series which ensures that the current solely can flow from drain to source. In the second mode, the current flows through the resistor RonBw and again, an ideal reverse blocking diode ensures that the current flows in the right direction. The body diode provides the current path for the third and the fourth mode. If the MOSFET is in on-state, the current will split between the reverse leg and the body diode as soon as the body diode's threshold voltage $V_{f}$ is reached. In off-state, the MOSFET is a simple diode conducting in reverse direction and blocking in forward direction.

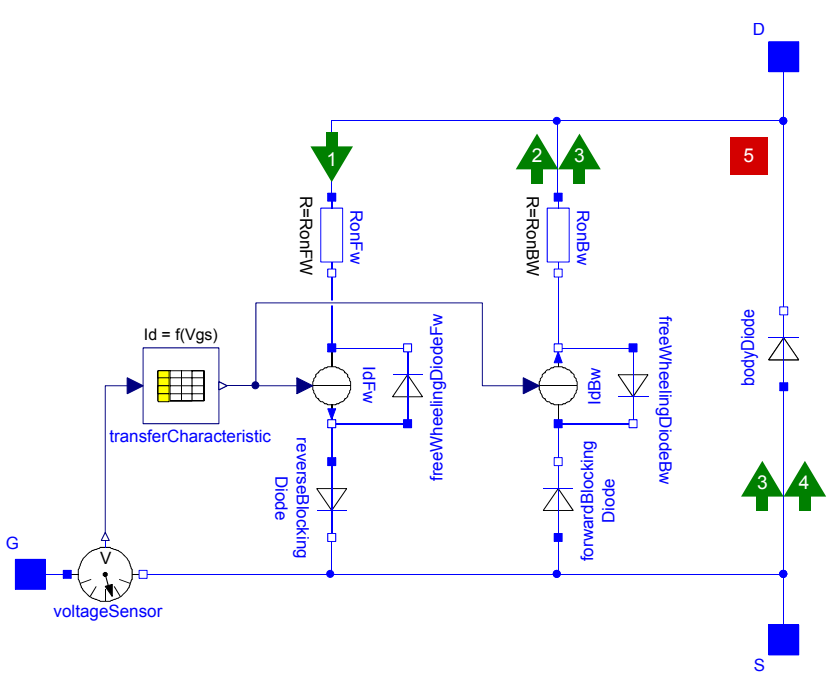

Figure 4: Static model of a power-MOSFET

\subsubsection{Dynamic Model}

For solving a demanding simulation task, a static model is often not sufficient enough. Especially when switching losses are of interest, a dynamic model is indispensable. The static model shall now be extended to introduce the component's dynamics, which are mainly an effect of capacitances between the MOSFET's connections. As the manufacturers do not provide these directly but in form of the input capacitance $C_{i s s}$, the output capacitance $C_{o s s}$ and the reverse trans- 
fer capacitance $C_{r s s}$, the following conversions have to be made to gain the effective capacitances between gate, drain and source:

$$
\begin{aligned}
C_{d s} & =C_{i s s}-C_{r s s} \\
C_{g d} & =C_{r s s} \\
C_{g s} & =C_{o s s}-C_{r s s}
\end{aligned}
$$

These equations are computed in the capTable block, which can be seen in the dynamic model in Figure 5. The characteristic curves $C_{i s s}=f\left(V_{g s}\right), C_{o s s}=$ $f\left(V_{g s}\right)$ and $C_{r s s}=f\left(V_{g s}\right)$, specified in the datasheet are stored inside tables. The computed values are the inputs to the signal-controlled capacitors, which can be seen in the model. The MOS-structure is modeled with an RC-circuit. The gate-source capacitor is charged through the internal gate resistor and the gate-source voltage is the voltage across the capacitor.

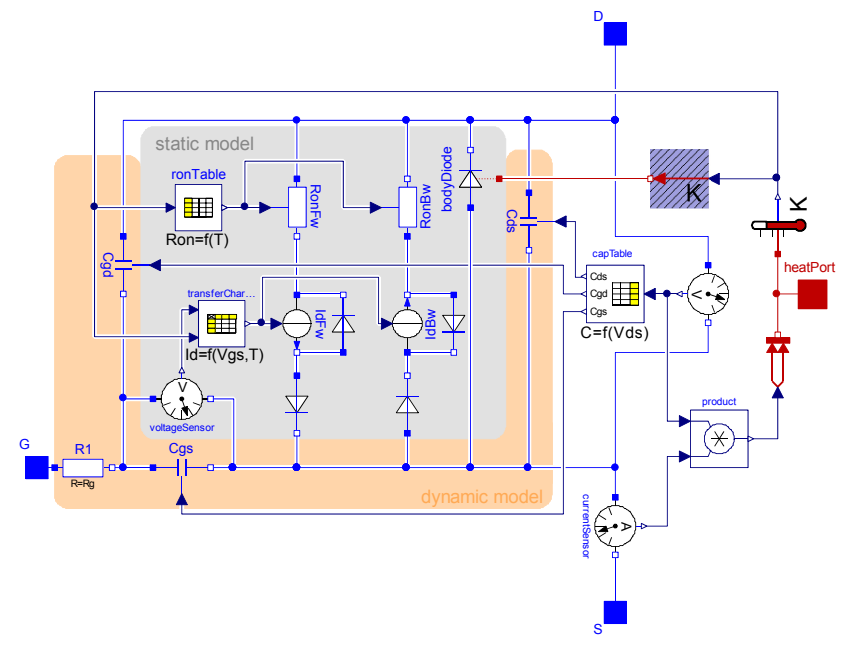

Figure 5: Dynamic model of a power-MOSFET

\subsubsection{Temperature Dependency}

Besides dynamics, temperature dependency is introduced to the model in Figure 5. Thus, in the transfer characteristic table a second input was introduced, i.e. $I_{d}=f\left(V_{g s}, T\right)$. The constant resistors have been replaced and are controlled by the characteristic curve $r_{o n}=f(T)$. The body diode model was extended such that it considers temperature in the diode's forward characteristic table $V_{f}=f\left(I_{f}, T\right)$ and that switching losses can be computed, i.e. the reverse recovery effect was modeled according to [3]. The total power losses are computed by multiplying the drain-source current and voltage and forwarded as heat flow to the heat port. This enables the use of a thermal network to compute the device's junction temperature.

\subsection{MOSFET Model Verification}

In order to verify the behavior of the static model in Figure 4, two test circuits have been created. For verifying modes 1, 2, 4 and 5, the circuit in Figure 6 is used. In order to test the on-state modes, the gate-source voltage is set to $10 \mathrm{~V}$; for testing the offstate modes it is set to $0 \mathrm{~V}$. The constant supply voltage source is either positive or negative depending on whether a forward conduction mode or a reverse conduction mode is tested. With the chosen load resistance, a current of approximately $100 \mathrm{~A}$ will be drawn.

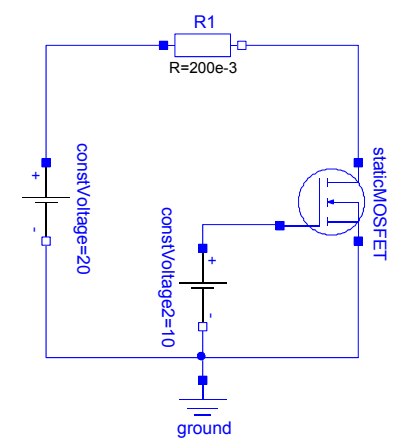

Figure 6: Test circuit for modes 1, 2, 4 and 5

Figure 7 shows the simulation results of the test circuit. It can be seen that in mode 1 , the total current flows in forward direction through the resistor RonFw. In mode 2, the input voltage is set negative and therefore the total current flows in the backward branch through the resistor RonBw. In the diagram of the fourth mode, it is shown that the current is neither flowing through RonFw nor through RonBw but through the body diode. The last diagram proves that no current at all flows in mode 5.
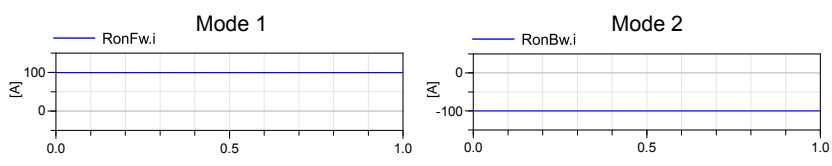

Mode 4

Mode 5
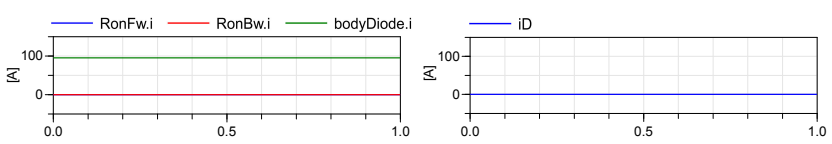

Figure 7: Simulation results for modes 1, 2, 4 and 5

To test the third static mode, the test circuit has been adapted in the way it is shown in Figure 8. The constant supply voltage source has been replaced by a signal-controlled voltage source with a ramp input. This causes the input voltage and therefore the current to increase linearly. 


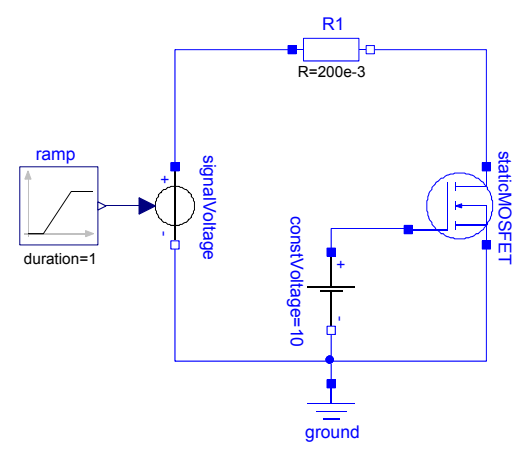

Figure 8: Test circuit for mode 3

Figure 9 shows the simulation result. The input voltage decreases from $-20 \mathrm{~V}$ to $-50 \mathrm{~V}$. At the beginning, the current flows in the backward branch through the resistor RonBw. At an input voltage level of about $-31 \mathrm{~V}$, the current through this resistor produces a voltage drop equal to the diode's threshold voltage. Hence, the diode starts conducting and builds a current divider together with RonBw. The total current is then divided into the two branches. The green curve represents the sum of the two branch currents, which is the total linearly decreasing current.

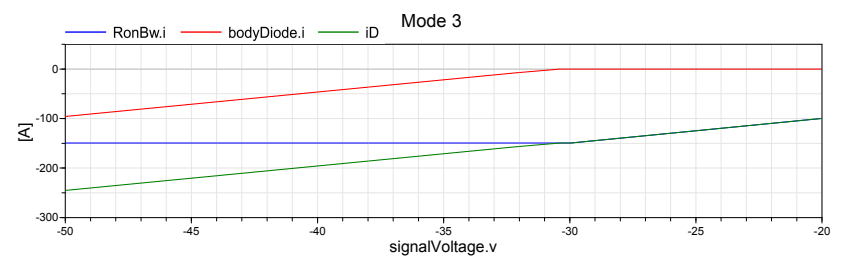

Figure 9: Simulation result for mode 3

The test circuit in Figure 10 is used to verify the MOSFET's dynamic behavior. The device is alternately turned on and off by the pulse voltage pattern applied between gate and source. A constant junction temperature is assumed and applied externally.

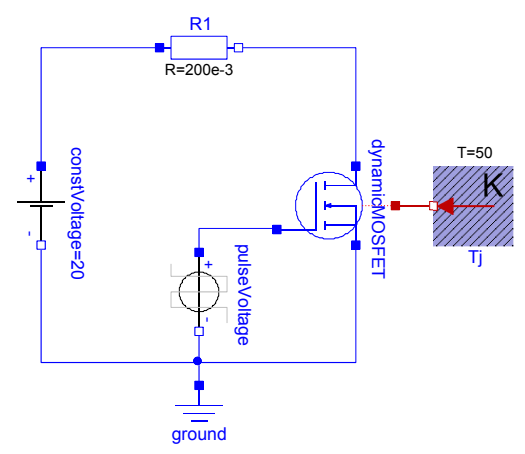

Figure 10: Test circuit dynamic behavior

Figure 11 depicts the simulation results. In the up- per diagram, one can see the drain current, the drainsource voltage and the power losses of the MOSFET. One can see the conduction losses and the turn-on and turn-off peaks whenever the device is switched. In the lower diagram, the switch-on behavior is shown more detailed.

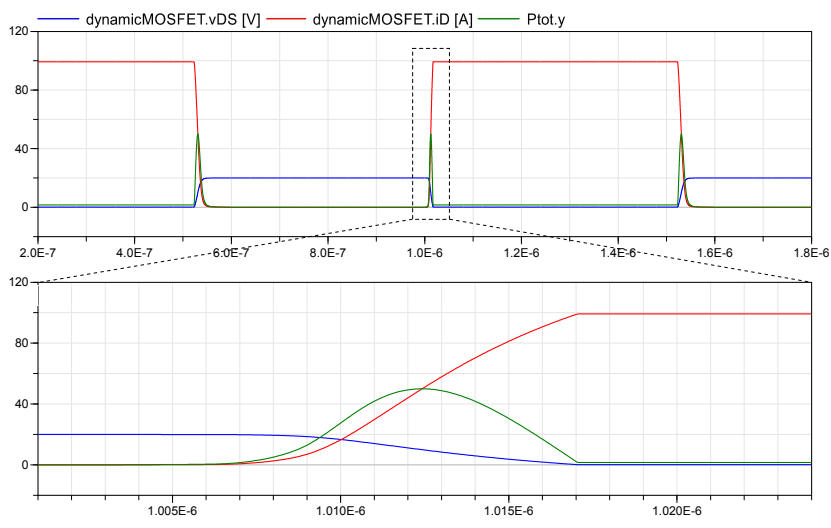

Figure 11: Switching waveforms and power losses

\subsection{MOSFET Model Validation}

The developed power-MOSFET model was validated with measurement data provided by a company producing electric drive systems. The data was obtained by measurements of a three-phase inverter of one of the company's drive systems. The circuit diagram of this inverter is shown in Figure 12. The device under test (DUT) is an Infineon IPB180N06S4-H1 powerMOSFET. Per phase leg, there are three high- and three low-side MOSFETs to be able to drive the desired load current. The company provided two spacevector modulation switching patterns for two different operating points of the electric machine and the corresponding power losses occurring per MOSFET. The data can be seen in Table 1:

Table 1: Operating points and loss power

\begin{tabular}{|c|c|c|c|}
\hline & RPM & Torque & Power \\
\hline Operating point 1 & $3700 \mathrm{rpm}$ & $3 \mathrm{Nm}$ & $1.1 \mathrm{~W}$ \\
\hline Operating point 2 & $700 \mathrm{rpm}$ & $3 \mathrm{Nm}$ & $0.9 \mathrm{~W}$ \\
\hline
\end{tabular}

Since a synchronous machine with three pole pairs is connected to the inverter, a mechanical revolution speed of $3700 \mathrm{rpm}$ results in an electrical frequency of $185 \mathrm{~Hz}$. In order to simulate the MOSFET's total power losses correctly, they have to be averaged over this frequency. The resistive-inductive load representing the electric machine, was parameterized in such a way that the measured current for the appropriate operating point is drawn. The three phase currents and the 


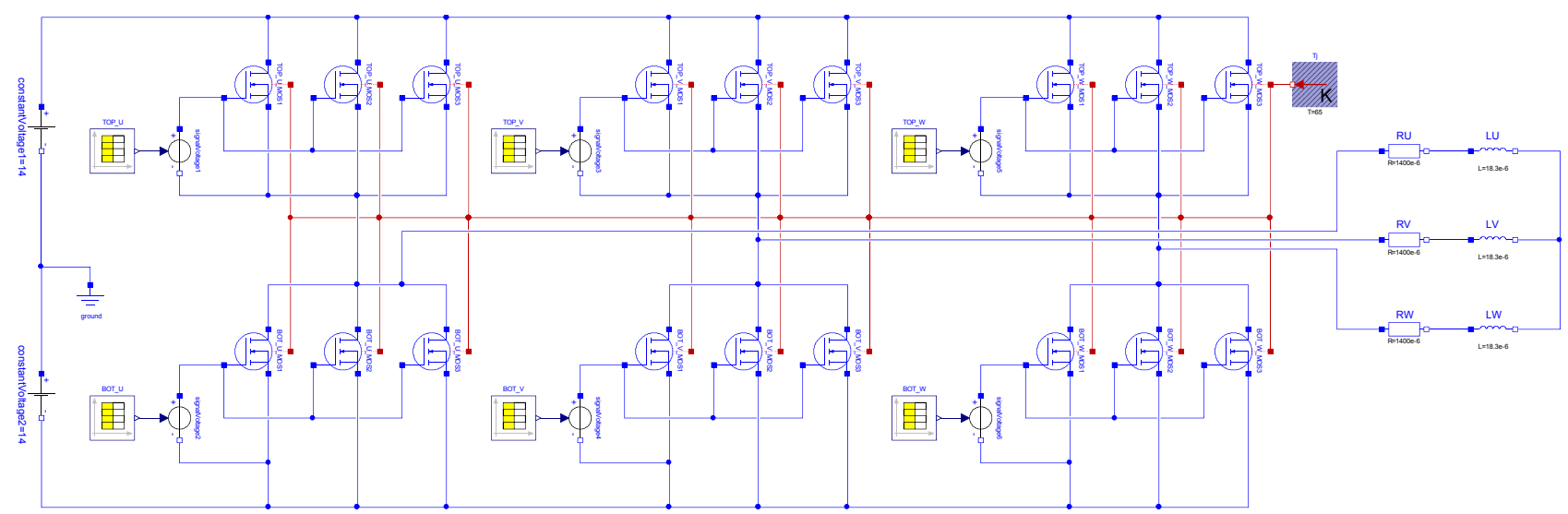

Figure 12: Power-MOSFET based three-phase inverter for driving an electric machine

average power losses per MOSFET are shown in Figure 13. The two curves in the lower diagram represent the power losses of a high- and a low-side MOSFET of the U-phase. The first average value can be computed after one period. It can be seen that once the U-phase current (blue signal in the upper plot) reaches steadystate, the average power losses over one period settle down at $1.12 W$.
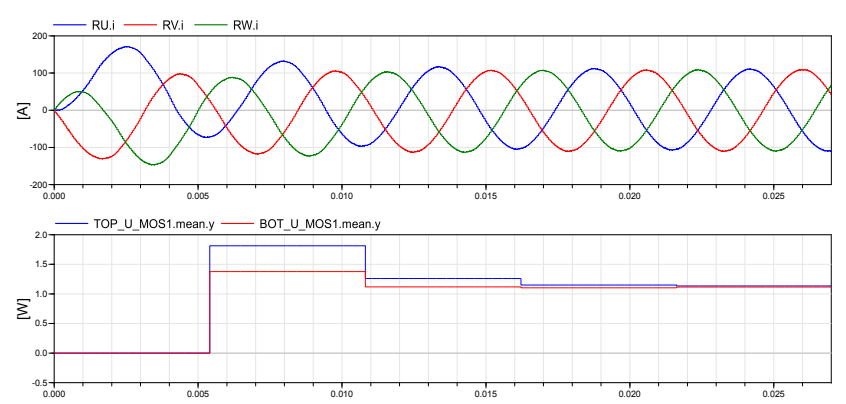

Figure 13: Simulation results of operating point 1

Figure 14 depicts the simulation results of the second operating point. At $700 \mathrm{rpm}$, the inverter's output frequency is $35 \mathrm{~Hz}$. Again, one can see the average power losses per MOSFET settle down in steady-state at $0.91 \mathrm{~W}$.
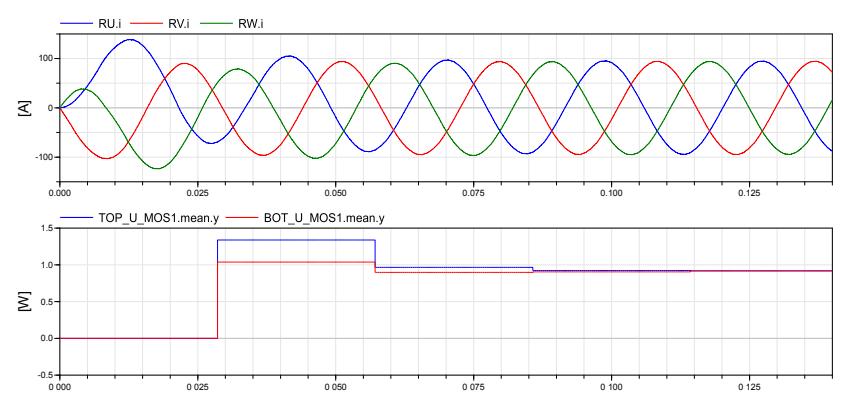

Figure 14: Simulation results of operating point 2
The relative error between the measured data and the simulation result lies within $2 \%$ in both operating points. These results speak especially for the behavioral modeling technique as well as for the accuracy of the provided information in the datasheets.

\section{Behavioral Modeling of IGBTs}

\subsection{IGBT Structure and its Modes of Opera- tion}

Concerning the IGBT technology, two different design principles are available, NPT- (non-punch through) and PT- (punch through) IGBTs. The basic structure of an NPT-IGBT is illustrated in Figure 15.

Contrary to power-MOSFETs, NPT-IGBTs have an additional $\mathrm{p}+$ doped layer between emitter and collector. This means that the forward characteristic of such a device does not behave like a classic resistance but more like a pn-junction. This pn-junction basically determines the IGBT's behavior in its forward and reverse mode.

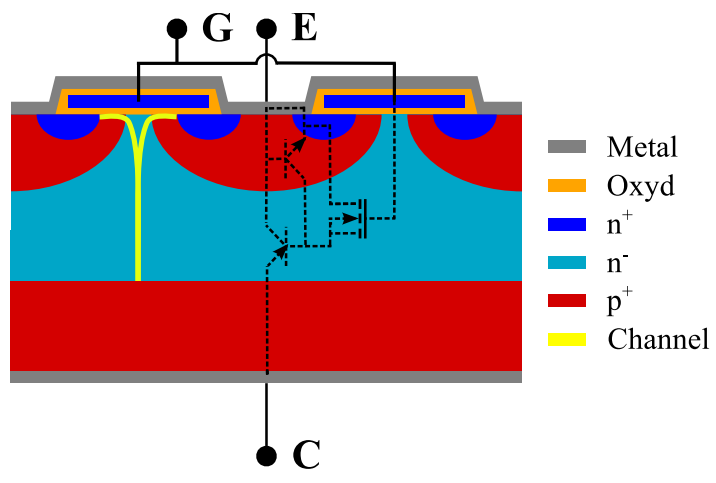

Source: Translated from [5]

Figure 15: Structure of a non-punch through IGBT 
If an additional $\mathrm{n}+$ doped layer is introduced between the p+ doped layer at the bottom and the ndoped layer, a PT structure is realized. Due to the additional $n+$ doped layer the shape of the electric field gets steeper which in turn allows for a smaller n- doped layer, i.e. reduces the chip size (refer to [7] for a more detailed description).

\subsection{IGBT Models}

A static and a dynamic behavioral model of an IGBT is introduced within this section, whereas the dynamic model is different for the NPT- and the PT-IGBT. The verification of the models is similar to the one of the MOSFET and is therefore not discussed in the paper. The validation of the models is currently under development.

\subsubsection{Static Model}

Figure 16 shows the static model of an IGBT. The output characteristic is modeled the same way as the diode's forward characteristic illustrated in Figure 1, i.e. the collector-emitter current is measured with a current sensor and fed into the table providing the IGBT's output characteristic $V_{C E}=f\left(I_{C}\right)$. The table's output is then fed into the signal-controlled voltage source. The transfer characteristic is modeled analogously to the MOSFET: The gate-emitter voltage is measured and the maximum current - provided by the transfer characteristic curve which is stored in a table - flows through the signal-controlled current source, i.e. it again behaves like a voltage controlled current source. As for the MOSFET model, a free-wheeling diode provides a path for the current which is supplied too much and a blocking diode ensures that the IGBT is not conducting in reverse direction.

\subsubsection{Dynamic NPT- and PT-IGBT Model}

To introduce dynamics to the model, three capacitors are introduced to the static model discussed before. The gate-emitter capacitor can be approximated as constant over the collector-emitter voltage (refer to Equation 1). The gate-collector capacitor - also known as Miller-Capacitance - strongly depends on the collector emitter voltage an can be approximated with Equation 2 [1, p. 15]. The input capacitor $C_{i s s}$ and the reverse transfer capacitor $C_{r e s}$ can be found in the datasheet.

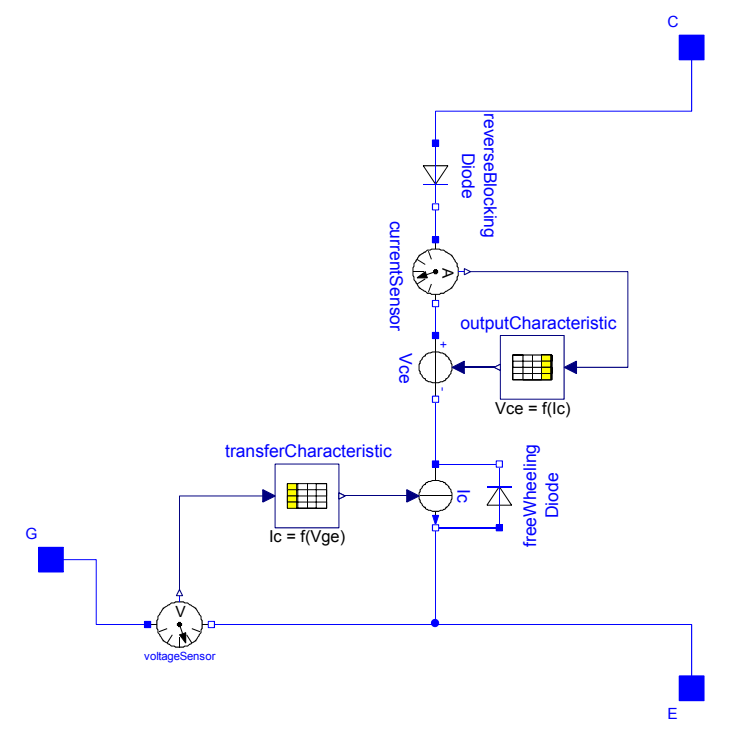

Figure 16: Static IGBT model

$$
\begin{aligned}
& C_{g e}\left(V_{c e}\right) \approx C_{i s s}(25 V)-C_{r s s}(25 V) \\
& C_{g c}\left(V_{c e}\right) \approx \frac{C_{r s s}(25 V) \cdot \sqrt{25 V}}{\sqrt{V_{c e}}}
\end{aligned}
$$

Where for instance $C_{i s s}(25 \mathrm{~V})$ and $C_{r s s}(25 \mathrm{~V})$ have been determined at a constant collector-emitter voltage of $25 \mathrm{~V}$.

Due to the internal semiconductor structure of PTIGBTs an additional output capacitor $C_{q}=f\left(V_{C E}\right)$ appears which is mainly responsible for the PT-IGBTs switching-off behavior (refer to [4]).

In Figure 17 the dynamic IGBT model is shown. The gate-emitter capacitor is charged over the internal gate resistance $R 1$. Equation 1 is implemented directly in the parameter window of $C_{g e}$ as $C_{i s s}$ and $C_{r e s}$ are input parameters to the model. Equation 2 is either implemented via equations or a table based model, i.e. the characteristic curve is derived by the equation. Again, temperature dependency has to be introduced to the model (shown for the output characteristic in Figure 17) and the power losses are forwarded as heat flow (not included in the model shown in Figure 17) to the heat port to allow the use of a thermal network.

The main problem of IGBT behavioral models is the lack of information in datasheets regarding tail current. Hence, the tail current has to be measured in advance and fed into the model. Alternatively, if there is no possibility to measure the tail current, the dynamic model can be replaced by the characteristic curves of the switching losses $E_{\text {on }}, E_{\text {off }}=f\left(I_{C}, T\right)$. 


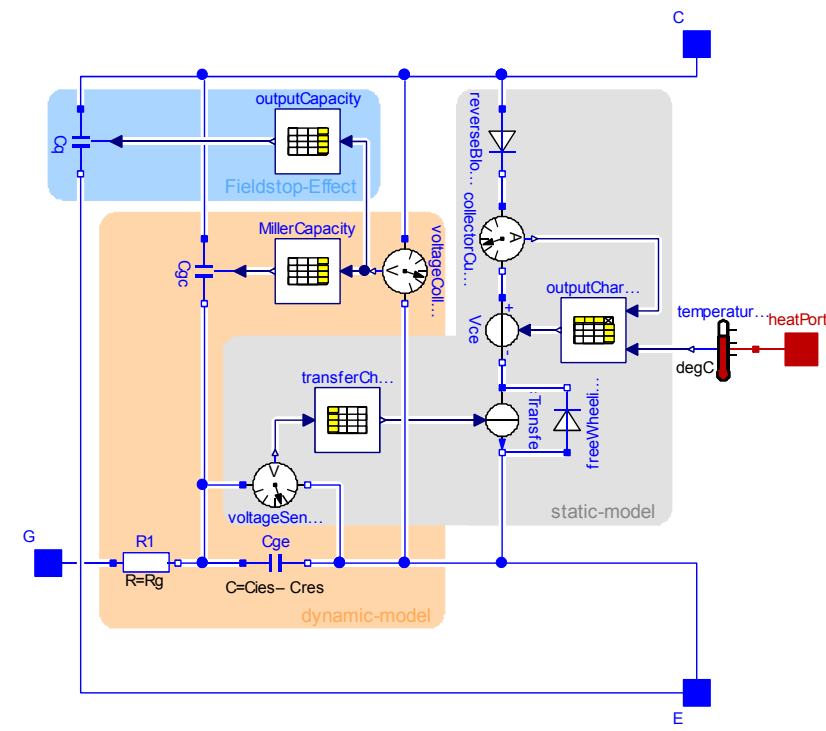

Figure 17: Dynamic PT-IGBT model. If the part highlighted in blue is removed, the model behaves like an NPT-IGBT

\section{Numerical Performance}

The simulation of power semiconductor components faces solvers with a whole series of challenges. To make statements about numerical stability and simulation time, the power-MOSFET model was tested in the three-phase inverter circuit in Figure 12 on its numerical performance ${ }^{1}$.

\subsection{Integration Algorithm}

Detailed modeling of switching operations usually results in so-called stiff systems, consisting of differential algebraic equations (DAE) having time constants that differ by several orders of magnitude, e.g. fast switching dynamics and slow thermal processes. These characteristics restrict the selection of the solver enormously. Only solvers based on implicit integration algorithms are suited for such simulations tasks.

Furthermore, switching operations lead to discontinuities in the simulation. Thereby it is distinguished between time- and state-events. In particular the latter causes the solver to iterate for the event, e.g. by using bi-section or regula-falsi algorithms, which increase the simulation time drastically. Even when using an implicit solver, it is not guaranteed that the iterations converge and the simulation succeeds. A deeper insight into this matter is given in [2]. Moreover, if the

\footnotetext{
1 any performance comparison was done on an Intel Core i53427U CPU, 1.8GHz, 8 GB RAM running Windows 8 (64Bit) and Dymola 2014
}

switching slopes are not treated ideally, the simulation performance becomes even worse, e.g. real switching slopes are in the region of several 100ns.

\subsection{Solver Settings}

In the upper part of Listing 1, default settings are given which basically match the Dymola standard settings except that the number of intervals is increased due to accuracy reasons. The simulation's performance results, which can be seen in the lower section of the listing, serve as reference values for any following comparison. The impact of the solver settings on the numerical performance is investigated on the basis of the number of function-, hessian- and jacobianevaluations. At this point it should be mentioned that the parameter for CPU integration time has to be interpreted with caution since it strongly depends on the utilization of the CPU and is therefore not precise in terms of repeatability.

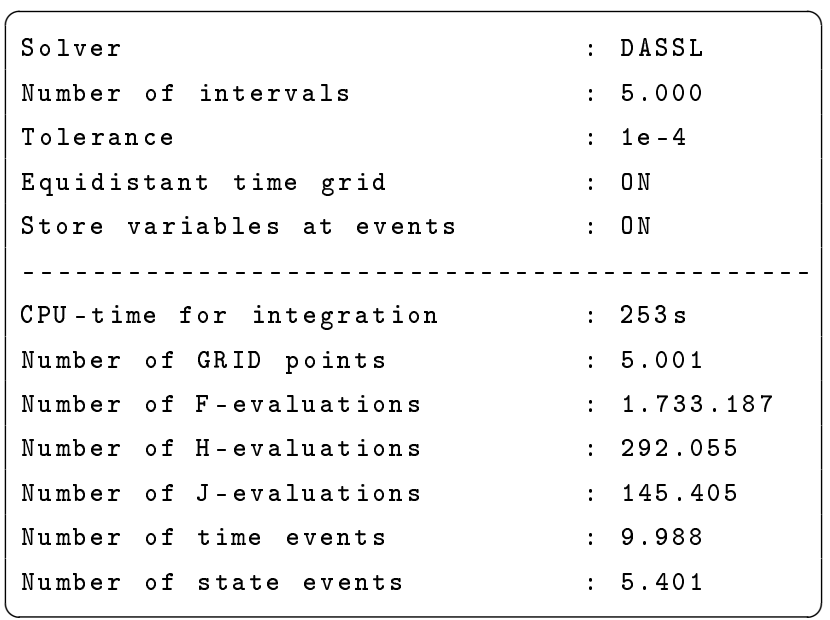

Listing 1: Default solver settings

First, the influence of the number of points plotted into the output diagram is investigated. If the equidistant time grid is deactivated ${ }^{2}$, the solver does not plot the computed values according to the number of intervals parameter, but instead it plots the entire computed values in the output diagram. The number of grid points then increases drastically (228.795) as it was expected whereas the number of $\mathrm{F}-, \mathrm{H}-$ and $\mathrm{J}$ evaluations basically stay the same. Nevertheless, the number of points that have to be plotted into the output diagram have a big influence on simulation time.

Next, the impact of the solver's integration tolerance is tested. In order to do this, the tolerance is increased by a power of ten. Due to less F-, H- and

\footnotetext{
${ }^{2}$ simulation setup - output tab - output selection
} 
J-evaluations, the simulation time could be decreased by approximately $25 \%$ but again resulting in a loss of accuracy. The same applies to the option 'store variables at events' ${ }^{3}$ when deactivated.

In summary, choosing the solver settings in order to optimize the simulation time strongly depends on how accurate the results shall be. The number of intervals should be chosen manually meaning that the equidistant time grid is activated.

\subsection{Optimization of the Model}

For optimizations of the model, first the ideal components are investigated. The model contains four ideal diodes. The on-resistance and off-conductance is set to $10^{-5} \Omega$ and $10^{-5} \mathrm{~S}$ by default. These values were changed to $10^{-7}$ for the next simulation. The results showed that this can be done without any problems regarding the simulation speed. A few more state events were generated because the system got stiffer. As a matter of fact, the smaller these values are chosen, the more accurate the power loss computation will be.

Also the tables have optimization potential because the interpolation-type influences the simulation performance. The user can choose between linear and continuous interpolation methods, whereas the first method decreases the simulation time slightly and at the same time is holding the number of additional events (due to the non-continuous method) in a negligible range.

Furthermore, the interpolations can be minimized by fitting polynomial functions through the characteristic curves stored in the tables. The challenge hereby is fitting the curves accurately while the order of the polynomial functions is held low. The problem with higher order polynomials is that they drift away drastically outside the fitted range and in terms of decreasing simulation time the opposite effect would occur. An approach hereby could be to split the curve in multiple sections and fit each with a separate polynomial function. When doing this, a polynomial of higher order can be split into multiple polynomials of lower order resulting in an invariant accuracy and a decrease of simulation time.

\subsection{Generating Table-Based Models for Sys- tem Simulation}

Although the simulation performance can be slightly improved, the models cannot be used in a system sim-

\footnotetext{
${ }^{3}$ simulation setup - output tab - output selection
}

ulation, e.g. to answer questions regarding temperature development or maximum driving range. For this purpose table-based models (efficiency maps) have to be provided, i.e. the overall losses at several operating points have to be stored in a table. Such models were already developed and verified for electric machines at Modelon $\mathrm{GmbH}$ and are currently developed for inverter models.

\section{Problems with Behavioral Models}

Among the bad numerical performance due to detailed description of the signals several other problems occur.

1. Firstly, datasheets provide values derived under nominal conditions. However, when such models are integrated in an electric powertrain the conditions strongly depend on the topology and are usually not as specified in the datasheet.

2. Secondly, values for parasitics elements are only available in datasheets of modules, e.g. inverter modules developed by the semiconductor manufacturer. The parasitics that occur in a specific layout have to be measured and afterwards integrated in the model (by introducing parasitic resistors, inductors and capacitors) to ensure that the switching behavior is modeled correctly.

3. Moreover, in case of IGBT models neither informations regarding tail current nor the output capacitor that appears in the PT structure are provided.

\section{Acknowledgments}

The models have been developed by our former student - Patrick Denz - during his thesis at Modelon GmbH. Especially the MOSFET model was modified by Patrick such that it covers all modes of operation. Many thanks for the great job at Modelon GmbH. Many thanks to Arendt Wintrich for the great support regarding state-of-the-art IGBT models.

\section{Conclusion}

The presented model of a power-MOSFET covers the static as well as the dynamic behavior of the component. Furthermore, it can be parameterized solely on the basis of characteristic curves and parameters specified in datasheets. The verification in various test circuits delivered reasonable results in static and dynamic 
applications. The model was validated with measurement data of a three-phase inverter motor drive system and the maximum relative error between the measured and simulated total power losses lies within $2 \%$.

Since behavioral models of power electronic components produce many events, the simulation becomes slow. It can be accelerated by widening up the integration tolerance, decreasing the number of intervals or not storing variables at events. Also optimizations on the model itself lead to a better simulation performance. However, when integrating the model into a simulation of the entire vehicle, it must be transformed into an efficiency map model which stores the power losses of different operating points in a table.

The IGBT models are way more complex since the tail current has to be measured in advance to model the signals correctly. If it is not possible - for whatever reason - to measure the tail current it is recommended to replace the dynamic model with tables storing the particular switching losses. This in turn increases the simulation performance and ensures that the losses are modeled correctly.

\section{References}

[1] Infineon Technologies AG. Automotive IGBT Module - Explanation of Technical Information. 2010.

[2] Francois E. Cellier and Ernesto Kofman. Continuous System Simulation. Springer, 2006.

[3] Alan Courtay. MAST Power Diode and Thyristor Models Including Automatic Parameter Extraction. CERN, European Laboratory for Particle Physics. Geneva, Switzerland, 1995.

[4] Bayerer Reinhold Heer, Daniel and Thomas Schütze. Trench Field-Stop IGBT3 Turn-Off. Infineon Technologies AG, Warstein, Germany, 2012.

[5] Josef Lutz. Halbleiter-Leistungsbauelemente: Physik, Eigenschaften, Zuverlässigkeit. Springer, 1st edition, 2006.

[6] Martin Otter, Hilding Elmqvist, and Sven Erik Mattsson. Modeling of mixed Continous/Discrete Systems in Modelica. 1999.

[7] Frank Pfirsch and Reinhold Bayerer. MOSgesteuerte Leistungsschalter: Konzepte und Schaltverhalten. VDE-Verlag, Bauelemente der Leistungselektronik und ihre Anwendungen, ETG-Fachtagung, 2006.
[8] Arendt Wintrich. Verhaltensmodellierung von Leistungshalbleitern für den rechnergestützten Entwurf leistungselektronischer Schaltungen. $\mathrm{PhD}$ thesis, Technische Universität Chemnitz, 1996.

[9] Arendt Wintrich, Ulrich Nicolai, Werner Tursky, and Tobias Reimann. Applikationshandbuch Leistungshalbleiter. ISLE, Ilmenau, 2010. 